\title{
Characterisation of calcium carbonate and its polymorphs from cockle shells (Anadara granosa).
}

\begin{abstract}
Calcium carbonate and its polymorphs from cockle shells (Anadara granosa) and commercial calcium carbonate were characterised using a variable pressure scanning electron microscopes (VPSEM), a transmission electron microscope (TEM), an energy dispersive Xray analyser (EDX), X-ray diffraction (XRD) and Fourier transmission infrared spectroscopy (FT-IR). Cubic-like calcite crystals of commercial calcium carbonate and rod-like aragonite crystals of cockle shell powders were observed by both SEM and TEM. The EDX results showed that the cockle shells contained more calcium and carbon than the commercial calcium carbonate, whereas the commercial calcium carbonate contained more oxygen than the cockle shells. FT-IR analyses revealed the presence of carbonate groups in both the cockle shells and the commercial calcium carbonate. FT-IR analyses also showed the presence of aragonite in the cockle shells and calcite in the commercial calcium carbonate. XRD analyses showed that the cockle shells powder contained aragonite, whereas the commercial calcium carbonate contained calcite. The cockle shells powder was formed with good quality calcium carbonate and contained calcium carbonate in the aragonite phase.
\end{abstract}

Keyword: Calcium carbonate; Polymorph; Cockle shell. 